\title{
Correction to: Notch Signaling and Human Papillomavirus-Associated Oral Tumorigenesis
}

Trinath Das, Rong Zhong, and Michael T. Spiotto

\section{Correction to:}

Chapter 8 in: J. Reichrath, S. Reichrath (eds.), Notch Signaling in Embryology and Cancer, Advances in Experimental Medicine and Biology 1287, https://doi.org/10.1007/978-3-030-55031-8_8

Unfortunately the book was published without correcting a typo in the author name in chapter 8 . The author name has been corrected now to read as follows:

Trinath Das 